\title{
Aspectos motores corporais e orais em um grupo de crianças com transtorno/atraso fonológico
}

\author{
Body and oral motor aspects in a group of children with \\ phonological delay/disorder
}

\author{
Ana Paula Ramos de Souza' ${ }^{1}$, Gabriela Lucas Pergher², Karina Carlesso Pagliarin ${ }^{3}$
}

\begin{abstract}
RESUMO
Objetivo: Comparar o desenvolvimento motor e aspectos orofaciais em crianças com transtorno e atraso fonológico. Métodos: Participaram da pesquisa 80 crianças de cinco a 11 anos de idade pertencentes à rede escolar regular de um município da Grande Porto Alegre com alterações fonológicas. Foi realizada uma entrevista com os pais através de um questionário semi-estruturado. Em seguida, foram avaliados o sistema estomatognático e a fala das crianças, e o diagnóstico de transtorno ou atraso fonológico foi confirmado para cada sujeito. Para a análise estatística, foram utilizados os testes de Fisher e o Qui-quadrado com nível de significância de 5\% ( $\mathrm{p}<0,05)$. Resultados: Não foram observadas diferenças estatisticamente significativas entre o grupo com atraso e o grupo com transtorno em todos os aspectos analisados. Conclusão: $\mathrm{O}$ atraso e o transtorno fonológico não apresentam distinção significativa em relação aos aspectos motores, orais, infecciosos e hábitos orais deletérios, demonstrando homogeneidade entre os grupos.
\end{abstract}

Descritores: Criança; Fala; Distúrbios da fala; Sistema estomatognático; Avaliação

\section{INTRODUÇÃO}

O distúrbio fonológico é caracterizado pela ininteligibilidade de fala como consequência de simplificações de regras fonológicas. Sua etiologia ainda é desconhecida, pelo fato de ser provavelmente multicausal, e pode ser encontrado em $10 \%$ da população pré-escolar e escolar, como uma das mais prevalentes inabilidades comunicativas nessa população ${ }^{(1)}$.

Uma pesquisa realizada ${ }^{(2)}$ enfocou a distinção entre o atraso e o transtorno através da fonologia autossegmental ${ }^{(3)}$. As características fonológicas do atraso são: presença de processos suprimidos na aquisição fonológica típica entre quatro e cinco anos de idade; isto é, quadro final de aquisição da líquida /r/, apagamentos de consoantes em finais de sílaba (/s/

Trabalho realizado no Curso de Fonoaudiologia da Universidade Luterana do Brasil - ULBRA - Canoas (RS), Brasil.

(1) Doutora, Professora do Curso de Fonoaudiologia e do Mestrado em Distúrbios da Comunicação Humana da Universidade Federal de Santa Maria - UFSM - Santa Maria (RS), Brasil.

(2) Especialista em Fonoaudiologia (ênfase em linguagem); Fonoaudióloga clínica - Porto Alegre (RS), Brasil.

(3) Pós-graduanda (Doutorado) em Psicologia da Pontifícia Universidade Católica do Rio Grande do Sul - PUC-RS - Porto Alegre (RS), Brasil; Professora do Curso de Graduação em Fonoaudiologia da Universidade Federal de Santa Maria - UFSM - Santa Maria (RS), Brasil.

Endereço para correspondência: Ana Paula Ramos de Souza. R. Raposo Tavares, 134/401, Medianeira, Santa Maria (RS), Brasil, CEP: 97015-560.

E-mail: ramos1964@uol.com.br

Recebido em: 26/2/2009; Aceito em: 10/9/2009 ou /r/), redução do encontro consonantal, e anteriorização de fricativas palatais. Já o transtorno se caracteriza pela presença de diversos processos, abrangendo muitas classes sonoras e com idiossincrasias, como a inversão na troca de traços (por exemplo: uma troca de ponto articulatório velar ou dorsal para alveolar é mais comum que o inverso, assim como a troca de uma líquida não-lateral para lateral é mais frequente que o contrário). Além disso, portadores de transtorno fonológico apresentariam trocas que levam à alteração de traços de raiz. Um exemplo seria a substituição de uma líquida como o /l/ por uma plosiva como o/t/ (/lua/ $\rightarrow$ [tua]), que evidencia a troca do traço [+soante] pelo traço [-soante], ou seja, uma alteração em um dos traços da raiz segmental.

$\mathrm{O}$ transtorno fonológico tem sido abordado em diversos estudos $^{(4-8)}$, sendo que alguns têm buscado o seu tratamento através da aplicação e comparação de modelos terapêuticos ${ }^{(5-7)}$; outros têm buscado investigar a etiologia do transtorno envolvendo o núcleo familiar ${ }^{(8-11)} \mathrm{e}$ até mesmo diferentes causas como, por exemplo, presença de otites ${ }^{(1,12)}$, de problemas respiratórios $^{(13)} \mathrm{e}$ de alterações do sistema estomatognático ${ }^{(14)}$. Porém, observou-se a necessidade de estudos considerando o desenvolvimento motor e orofacial de crianças com transtornos fonológicos, com o intuito de averiguar possíveis interferências fonéticas em tais distúrbios.

Tendo isto em vista, este trabalho teve como objetivo comparar o desenvolvimento motor e aspectos orofaciais em crianças com transtorno e atraso fonológico pertencentes à rede escolar regular de um município da grande Porto Alegre. 


\section{MÉTODOS}

Este estudo é do tipo observacional, descritivo, transversal, histórico, vinculado a um projeto devidamente registrado no Comitê de Ética em Pesquisa (CEP) da Universidade Luterana do Brasil (ULBRA) sob n ${ }^{\circ} 107 / 2002$. Para que os sujeitos participassem deste estudo, os pais ou responsáveis assinaram o Termo de Consentimento Livre e Esclarecido, autorizando a realização da pesquisa e publicações dos resultados. Também responderam a um questionário semi-estruturado com questões abertas e fechadas para maiores informações sobre o desenvolvimento e evolução orofacial de seus filhos.

Os critérios para seleção da amostra obedeceram à categorização exposta a seguir. Foram excluídas crianças sindrômicas, com distúrbios de linguagem, com alterações auditivas ou neurológicas ou com algum outro tipo de alteração que não as caracterizasse como tendo inteligência e desenvolvimento normais para a idade. Tomou-se a observação do professor e dos pesquisadores a respeito dos comportamentos das crianças como forma de exclusão em casos em que houvesse qualquer dúvida em relação à normalidade intelectual. Todos os sujeitos foram submetidos à inspeção do meato acústico externo e à triagem auditiva para descartar alterações auditivas tanto condutivas quanto neurossensoriais.

A amostra foi composta por 80 crianças, de cinco a 11 anos, pertencentes às escolas municipais de uma cidade da Grande Porto Alegre, RS, de ambos os gêneros.

A coleta dos dados foi realizada em 2004. Na ocasião, foi criado um banco de dados a partir do qual foram analisadas as variáveis desenvolvimento motor, uso de mamadeira, uso de chupeta e presença de hábitos orais deletérios, de doenças otorrinolaringológicas, respiração oral, avaliação das praxias orais, entre estas as de lábios e língua.

Os dados obtidos com relação ao desenvolvimento motor referiam-se à idade em que a criança firmou a cabeça (controle cefálico, cujo parâmetro normal foi considerado até quatro meses), em que sentou (controle de tronco, parâmetro de normalidade considerado até sete meses), em que engatinhou (considerado não atrasado até 12 meses) e em que andou (parâmetro de normalidade considerado até um ano e seis meses).

Questionaram-se os pais se as crianças fizeram uso de mamadeira e chupeta (além dos dois anos de idade) ou se apresentavam algum hábito deletério como roer unhas ou chupar dedos.

Quanto às doenças otorrinolaringológicas, verificou-se se havia ocorrência de infecções de vias aéreas superiores (otites, faringite ou amigdalites, rinite ou sinusite) frequentes. Foram utilizados como parâmetro para afirmar a ocorrência de infecções de repetição o número superior a três vezes ao ano.

$\mathrm{Na}$ avaliação do sistema estomatognático, foram avaliados aspectos como a morfologia da face, tonicidade, formato, postura e mobilidade dos órgãos fonoarticulatórios (lábios, língua, palato mole e duro, dentes, bochechas e mandíbula). Deu-se ênfase principalmente aos movimentos de língua (afilar, alargar, elevar, retrair, estalar, baixar, projetar, lateralizar e vibrar) e lábios (protruir, vibrar, fazer bico, lateralizar bico, soprar e apertar). Também foram verificadas as funções de respiração (nasal, oral e mista), da mastigação e da deglutição.
Foi realizada observação informal da linguagem compreensiva e expressiva por meio de conversa espontânea e observou-se a adequação das respostas, organização lógica do pensamento, estrutura gramatical das sentenças e o vocabulário empregado.

Para confirmar o diagnóstico de transtorno ou atraso, utilizou-se procedimento de coleta a partir da nomeação espontânea de figuras - Avaliação Fonológica da Criança ${ }^{(15)}$ - sendo os dados transcritos foneticamente e analisados os processos existentes.

A partir da identificação de processos, os sujeitos foram classificados como tendo atraso ou transtorno fonológico a partir dos critérios utilizados em estudo linguístico ${ }^{(3)}$. Os critérios para classificação como atraso foram: presença de processos suprimidos na aquisição fonológica típica entre quatro anos e cinco anos, ausência de inversão na troca de traços usualmente alterados na aquisição fonológica típica, ausência de alteração de traços de classe principal (traços de raiz). Os sujeitos deste grupo apresentaram alterações nas fricativas palatais e líquidas e na estrutura CVC e CCV. Os critérios para classificação como transtorno fonológico incluíram: presença de processos cronologicamente anteriores aos encontrados entre quatro e cinco anos em co-ocorrência aos típicos desta faixa etária, inversão na troca de traços usualmente alterados na aquisição fonológica típica, presença de processos idiossincráticos abrangendo a troca de traços de raiz (por exemplo [+soante] [-soante], [1] [t]). Os sujeitos apresentaram sistemas fonológicos bastante defasados em relação ao sistema padrão de crianças mais jovens com desenvolvimento normal.

Dos 80 sujeitos selecionados para pesquisa, 56 apresentavam atraso fonológico - 30\% do gênero feminino e $70 \%$ do masculino -, e 24 apresentavam transtorno fonológico, sendo $33 \%$ do gênero feminino e $67 \%$ do masculino.

A partir da identificação dos grupos, foram quantificados os resultados para cada uma das variáveis estudadas em ambos os grupos. Para a análise da comparação dos dados entre o grupo que apresentou atraso fonológico e o grupo que apresentou transtorno fonológico, foram utilizados os testes de Fisher e o Qui-quadrado, realizados através do software SPSS versão 10.0, e o nível de significância adotado foi de $5 \%(\mathrm{p}<0,05)$.

\section{RESULTADOS}

Para este artigo, demonstram-se os resultados que foram mais importantes na análise: movimentos de língua e lábios, presença de respiração oral, hábitos deletérios, prolongamento do uso da mamadeira ou chupeta, presença de otites de repetição, faringites ou amigdalites, rinite ou sinusite e evolução motora (controle cefálico, sentar, engatinhar, andar).

Na Tabela 1 está representada a percentagem de sujeitos com atraso e transtorno fonológico que apresentaram demora em adquirir o controle cefálico, para sentar, para engatinhar e para caminhar. Nesta tabela também se encontram os resultados da análise estatística realizada, a fim de verificar se houve diferença estatisticamente significante entre os grupos.

Pode-se perceber que não houve diferença estatisticamente significante entre os grupos em nenhum dos aspectos analisados quanto ao desenvolvimento motor. Porém observa-se que 
Tabela 1. Porcentagem de crianças com atraso e transtorno fonológico com desenvolvimento motor atrasado

\begin{tabular}{lcccc}
\hline Grupo & \multicolumn{4}{c}{ Desenvolvimento motor atrasado } \\
\cline { 2 - 5 } & Cefálico & Sentar & Engatinhar & Caminhar \\
\hline Atraso & $43,8 \%$ & $25,5 \%$ & $2,3 \%$ & $7,5 \%$ \\
Transtorno & $50,0 \%$ & $18,2 \%$ & $5,0 \%$ & $13,0 \%$ \\
\hline Valor de $p$ & 0,792 & 0,562 & 0,531 & 0,426 \\
\hline
\end{tabular}

Teste exato de Fisher

os sujeitos com transtorno fonológico precisaram de um tempo maior para começar a firmar a cabeça, engatinhar e caminhar do que as crianças com atraso fonológico.

Na Tabela 2, está representada a percentagem de sujeitos com atraso e com transtorno fonológico que fizeram uso de chupeta, mamadeira ou possuíam algum hábito oral deletério ou ainda que apresentavam respiração oral. Nesta tabela também se encontram os resultados da análise estatística realizada, a fim de verificar se houve diferença estatisticamente significante entre os grupos.

Tabela 2. Hábitos orais dos sujeitos com atraso e transtorno fonológico

\begin{tabular}{lcccc}
\hline Grupo & Chupeta & Mamadeira & $\begin{array}{c}\text { Respiração } \\
\text { oral }\end{array}$ & $\begin{array}{c}\text { Hábitos } \\
\text { deletérios }\end{array}$ \\
\hline Atraso & $71,2 \%$ & $96,0 \%$ & $26,79 \%$ & $70,6 \%$ \\
Transtorno & $68,4 \%$ & $90,0 \%$ & $25,00 \%$ & $81,8 \%$ \\
\hline Valor de $\mathrm{p}$ & 1,000 & 0,572 & 1,000 & 0,699 \\
\hline
\end{tabular}

Teste exato de Fisher

A partir da análise estatística, observa-se que não houve diferença significativa entre os grupos nos aspectos analisados. Apesar disso, verifica-se que no grupo com atraso fonológico observou-se maior uso de chupeta e mamadeira e maior ocorrência de respiração oral; porém, o grupo que apresentou maior incidência de hábitos deletérios foi o com transtorno fonológico.

A Tabela 3 refere-se à percentagem de sujeitos com atraso e com transtorno fonológico que apresentaram histórico de otites, rinites/sinusites e amigdalites/faringites frequentes. Nesta tabela também se encontram os resultados da análise estatística realizada, a fim de verificar se houve diferença estatisticamente significativa entre os grupos.

Tabela 3. Alterações de vias aéreas superiores em crianças com atraso e com transtorno fonológico

\begin{tabular}{lccc}
\hline Grupo & Rinite/sinusite & Otite & $\begin{array}{c}\text { Amigdalite/ } \\
\text { faringite }\end{array}$ \\
\hline Atraso & $13,00 \%$ & $23,40 \%$ & $16,00 \%$ \\
Transtorno & $15,80 \%$ & $10,50 \%$ & $35,00 \%$ \\
\hline Valor de $p$ & 0,714 & 0,318 & 0,109 \\
\hline
\end{tabular}

Teste exato de Fisher

Pode-se verificar que não houve diferença estatisticamente significativa entre os grupos em relação aos aspectos analisados. Observa-se ainda que rinites/sinusites e amigdalites/ faringites foram mais frequentes no grupo com transtorno fonológico, já as otites foram mais frequentes no grupo com atraso.

Na Tabela 4 estão representadas as porcentagens de sujeitos com atraso e transtorno fonológico com e sem alterações nos movimentos de lábios e língua. Nesta tabela também se encontram os resultados da análise estatística realizada.

Tabela 4. Mobilidade de língua em crianças com atraso e com transtorno fonológico

\begin{tabular}{lcccc}
\hline \multirow{2}{*}{ Grupo } & \multicolumn{2}{c}{ Lábios } & \multicolumn{2}{c}{ Língua } \\
\cline { 2 - 5 } & $\begin{array}{c}\text { Com } \\
\text { alterações }\end{array}$ & $\begin{array}{c}\text { Sem } \\
\text { alterações }\end{array}$ & $\begin{array}{c}\text { Com } \\
\text { alteraçòes }\end{array}$ & $\begin{array}{c}\text { Sem } \\
\text { alterações }\end{array}$ \\
\hline Atraso & $23,20 \%$ & $76,80 \%$ & $30,40 \%$ & $69,60 \%$ \\
Transtorno & $33,30 \%$ & $66,70 \%$ & $33,30 \%$ & $66,70 \%$ \\
\hline Valor de $p$ & \multicolumn{2}{c}{0,409} & \multicolumn{2}{c}{0,798} \\
\hline
\end{tabular}

Teste Qui-quadrado

Quanto às praxias de lábios e língua dos 80 sujeitos, 59 $(73,80 \%)$, tanto com atraso como com transtorno, não apresentaram alterações nos movimentos de lábios, o que também pode ser observado para os movimentos de língua, em que 55 $(68,80 \%)$ sujeitos obtiveram bom desempenho. Entretanto, verifica-se que o grupo com transtorno fonológico apresentou maior número de indivíduos com dificuldades na execução dos movimentos de lábios e língua que o grupo com atraso. Não houve associação estatisticamente significativa entre os grupos nos aspectos analisados.

\section{DISCUSSÃO}

Os resultados apresentados na Tabela 1 mostram que metade dos sujeitos com transtorno fonológico apresenta controle cefálico tardio, e, no grupo com atraso, este índice também é alto, 43,80\%. Observou-se, na aplicação dos questionários, que tal resposta era muito variada entre as mães e, em alguns casos, até incoerente com as idades atribuídas entre as demais habilidades motoras corporais investigadas. A hipótese que se sugere para este dado é a de que a lembrança das habilidades motoras mais remotas vai-se perdendo com o passar dos anos. A lembrança do caminhar parece ser um dado mais recente e mais confiável em termos percentuais, pois não se espera que sujeitos com transtorno ou atraso fonológico apresentem déficits corporais importantes pela própria caracterização clínica desta patologia ${ }^{(4-8)}$. O percentual próximo de $10 \%$ de atraso no caminhar sugere que possa haver um pequeno subgrupo com transtorno ou atraso fonológico ao qual se associam dificuldades motoras corporais. Isso sugere a possibilidade de haver alterações fonéticas associadas às fonológicas, já que imaturidades psicomotoras evolutivas podem estar relacionadas a problemas de fala, como ocorre nas dispraxias verbais versus corporais ${ }^{(16)}$.

A partir dos resultados apresentados na Tabela 2, verifica-se a ocorrência do uso de mamadeira e chupeta e de hábitos orais deletérios e respiração oral. Pode-se observar que a maioria dos sujeitos analisados (80\%), tanto com atraso como com transtorno, fizeram uso de mamadeira além dos dois anos de idade. Resultado semelhante foi encontrado em uma pesquisa ${ }^{(17)} \mathrm{em}$ 
que quase a totalidade das crianças com idades entre quatro e seis anos apresentaram este hábito por mais de 36 meses. Esse fato sugere que essa questão de hábitos orais deletérios é aspecto geral da população, possivelmente indicando uma mudança de um aspecto cultural do cuidado com a criança que deve preocupar os profissionais de saúde que trabalham com a morfologia e as funções orais, e também com a fala.

Estudos ${ }^{(18,19)}$ afirmam que o uso prolongado de mamadeira e chupeta e hábitos orais como sucção digital, por exemplo, podem trazer danos orais como problemas na fala, na oclusão dentária, na musculatura da face e na respiração oral.

Pode-se observar ainda que poucos sujeitos com atraso (15 sujeitos) e com transtorno (seis sujeitos) apresentaram respiração oral, concordando com os achados de um estudo ${ }^{(14)}$ em que foi observada maior ocorrência de respiração nasal em sujeitos com transtorno fonológico do que respiração oral.

De acordo com os resultados apresentados na Tabela 3, observa-se a baixa ocorrência de infecções de vias aéreas superiores em crianças com transtorno e com atraso fonológico.

Em uma pesquisa ${ }^{(1)}$, as autoras caracterizaram as semelhanças entre os sujeitos com transtorno fonológico e constataram, em sua amostra, que $80 \%$ das crianças apresentavam histórias de otites. Na presente pesquisa, o número de crianças com este histórico não foi tão significante, embora sua importância para problemas de fala e linguagem não possa ser descartada.

Com relação às infecções de vias aéreas superiores, a mesma pesquisa ${ }^{(1)}$ observou que geralmente crianças com transtorno fonológico apresentam história de alterações de vias aéreas superiores, contrapondo-se aos achados deste estudo.

Em um estudo mais recente ${ }^{(12)}$, com o objetivo de verificar os tipos de ocorrência de uso de processos fonológicos em crianças com transtorno fonológico, com e sem histórico de otite média, as autoras observaram que, apesar da otite média ter relação com o transtorno fonológico, não houve diferenças entre os grupos, com e sem otites, em relação aos processos fonológicos apresentados. Esse resultado parece estar relacionado com o resultado desta pesquisa, na medida em que não houve diferença entre o grupo com atraso e transtorno, ambos com tipologias distintas de processos fonológicos ${ }^{(2)}$.

A partir dos dados apresentados na Tabela 4, verifica-se que a maioria dos sujeitos não apresentou alterações nos movimentos de lábios, discordando de um estudo ${ }^{(14)}$ semelhante, que verificou que, para a maioria dos sujeitos com transtorno, houve alteração de mobilidade labial.

Quanto à mobilidade de língua, pode-se observar que a maioria dos sujeitos com transtorno e atraso fonológico não apresentaram dificuldades para realização dos movimentos solicitados. Tais achados discordam dos resultados encontrados em um estudo ${ }^{(14)}$ em que as autoras verificaram alterações na mobilidade de língua em um maior número de sujeitos com transtorno fonológico.

Em um trabalho ${ }^{(20)}$ sobre praxias linguais em relação à produção do /r/ fraco na infância, realizado com um grupo de dez crianças que produziam corretamente o fonema /r/ e o outro grupo de crianças que não produziam o fonema, as autoras encontraram crianças que não apresentaram algumas das praxias linguais no grupo que não produzia o fonema. Esse fato não se confirmou neste estudo, fortalecendo a hipótese de que este grupo foi razoavelmente homogêneo, em sua maioria, em relação a ter exclusivamente um atraso ou transtorno fonológico e não fonético.

Esse estudo se faz importante, pois os aspectos analisados poderiam auxiliar na prática clínica do profissional fonoaudiólogo tanto no momento diagnóstico como no planejamento terapêutico, facilitando, dessa maneira, a intervenção. Entretanto esta pesquisa confirma os achados da literatura quanto à presença de alterações na fala de algumas crianças e na ausência de fatores orgânicos identificáveis ${ }^{(12)}$.

\section{CONCLUSÃO}

De um modo geral, os resultados da pesquisa demonstram que a distinção entre atraso e transtorno fonológico não se concretizou como significativa entre aspectos motores corporais e orais, apresentando uma homogeneidade entre ambos os grupos nesses aspectos. Do mesmo modo, isso pôde ser verificado em relação a processos infecciosos, em vias aéreas superiores, e em relação a hábitos orais deletérios. Portanto, tal distinção parece ficar no âmbito clínico de linguagem de modo exclusivo, sugerindo que a mesma não se justifica de um ponto de vista clínico mais geral.

Sugere-se a continuidade da investigação de grupos com tal distinção, a abordagem de outros aspectos evolutivos, e, sobretudo, a respeito da intervenção e da evolução terapêutica, para observar se a diferença se faz relevante enquanto distinção de subgrupos dentro da patologia fonológica infantil.

\begin{abstract}
Purpose: To compare the motor development and orofacial aspects in children with phonological disorder and delay. Methods: The participants were 80 children with phonological deficits and ages ranging from five to 11 years, who belonged to the regular school system of a city in Rio Grande do Sul, Brazil. An interview with their parents was conducted, using a semi-structured questionnaire. After that, it was carried out the assessment of the subjects' stomatognathic system and speech, and the diagnosis of phonological delay or disorder was confirmed. The Chi-square test and the Fisher test were used for statistical analysis, with significance level of $5 \%$ ( $<<0,05)$. Results: There were no statistically significant differences between the group with phonological delay and the group with phonological disorder in all aspects examined. Conclusion: Phonological delay and disorder do not show significant distinction regarding motor, oral and infectious aspects, together with deleterious oral habits, as shown by the homogeneity between the groups.
\end{abstract}

Keywords: Child; Speech; Speech disorders; Stomatognathic system; Evaluation 


\section{REFERÊNCIAS}

1. Wertzner HF, Oliveira MMF. Semelhanças entre os sujeitos com distúrbio fonológico. Pró-Fono. 2002;14(2):143-52.

2. Ramos APF, Carreirao, L; Pergher, GL, Collares, LM; Marques, J Distúrbios fonológicos: perfil fonológico e inteligibilidade de fala. Cad Pesq Ling. 2005;1(1):67-78.

3. Lamprecht R. A aquisição fonológica normal e com desvios fonológicos evolutivos: aspectos quanto à natureza da diferença. Letras Hoje. 1995;30(4):117-25.

4. Wertzner HF, Papp ACCS, Amaro L, Galea DES. Relação entre os processos fonológicos e classificação perceptiva de inteligibilidade de fala no transtorno fonológico. Rev Soc Bras Fonoaudiol. 2005;10(4):193-200.

5. Crosbie S, Holm A, Dodd B. Intervention for children with severe speech disorder: a comparison of two approaches. Int J Lang Commun Disord. 2005;40(4):467-91.

6. Mota HB, Keske-Soares M, Bagetti T, Ceron MI, Melo Filha MGC. Análise comparativa da eficiência de três diferentes modelos de terapia fonológica. Pró-Fono. 2007;19(1):67-74.

7. Keske-Soares M, Brancalioni AR, Marini C, Pagliarin KC, Ceron MI. Eficácia da terapia para desvios fonológicos com diferentes modelos terapêuticos. Pró-Fono. 2008;20(3):153-8.

8. Dodd B. Evaluation of core vocabulary intervention for treatment of inconsistent phonological disorder: three treatment case studies. Child Lang Teach Ther. 2008;24(3):307-27.

9. Kovas Y, Hayiou-Thomas ME, Oliver B, Dale PS, Bishop DV, Plomin $R$. Genetic influences in different aspects of language development: the etiology of language skills in 4.5-year-old twins. Child Dev. 2005;76(3):632-51.

10. Weber DE, Vares MA, Mota HB, Keske-Soares M. Desenvolvimento do sistema fonológico de gêmeos monozigóticos com desvio fonológico: correlação a fatores genéticos e ambientais. Rev CEFAC. 2007;9(1):329.
11. Lewis BA, Freebairn LA, Hansen AJ, Miscimarra L, Iyengar SK, Taylor HG. Speech and language skills of parents of children with speech sound disorders. Am J Speech Lang Pathol. 2007;16(2):108-18.

12. Wertzner HF, Pagan LO, Galea DES, Papp, ACCS. Características fonológicas de crianças com transtorno fonologico com e sem histórico de otite média. Rev Soc Bras Fonoaudiol. 2007;12(1):41-7.

13. Wertzner HF, Rosal CAR, Pagan LO. Ocorrência de otite média e infecções de vias aéreas superiores em crianças com distúrbio fonológico. Rev Soc Bras Fonoaudiol. 2002;7(1):31-7.

14. Casarin MT, Gindri G, Keske-Soares M, Mota HB. Alterações do sistema estomatognático em distúrbios da fala. Rev Soc Bras Fonoaudiol. 2006;11(4):223-30.

15. Yavas M, Hernandorena CLM, Lamprecht RR. Avaliação fonológica da criança: reeducação e terapia. Porto Alegre: Artes Médicas; 1991.

16. Forrest, K. Diagnostic criteria of developmental apraxia of speech used by clinical speech-language pathologists. Am J Speech Lang Pathol. 2003;12(3):376-80.

17. Galvão ACUR, Menezes SFL, Nemr K. Correlação de hábitos orais deletérios entre crianças de 4:00 e 6:00 anos de escola pública e escola particular da cidade de Manaus- AM. Rev CEFAC. 2006;8(3):328-36.

18. Pastor I, Montana K. Amamentação natural no desenvolvimento do sistema estomatognático. Rev Odontopediat. 1994;3(4):185-91.

19. Cavassani VGS, Ribeiro S, Nemr NK, Greco AM, Köhle J, Lehn C. Hábitos orais de sucção: estudo piloto em população de baixa renda. Rev Bras Otorrinolaringol. 2003;69(1):106-10.

20. Fonseca RP, Dornelles S, Ramos APF. Relação entre a produção do r-fraco e as praxias linguais na infância. Pró-Fono. 2003;15(3):229-40. 